consider a slight modification to their application so that it includes the species with which the researcher has had prior experience. The IACUC protocol in question should also be flagged for post approval monitoring (PAM) to determine what factors may have led to the death of the first 5 mice. The PAM process can not only help with the surgical procedure itself but can also shed some light on other perioperative elements (e.g. surgical preparation, anesthesia, recovery, analgesia, etc.)
These steps taken by both the IACUC and Schwartz will improve the quality of the research being conducted while also helping to refine the process of assessing the required training at the institution ${ }^{2}$. These steps can also serve to enhance the trust between researchers and the IACUC which is in the best interest of the animals, the institution, and the research.

James B. Finlay* and Aimee Buehler City of Hope - Beckman Research Institute,
Duarte, CA, USA.

*e-mail: jfinlay@coh.org

Published online: 23 October 2019

https://doi.org/10.1038/s41684-019-0418-3

\section{References}

1. Institute for Laboratory Animal Research. Guide for the Care and Use of Laboratory Animals 8th edn. (National Academies Press, Washington, DC, 2011).

2. Public Health Service. Policy on Humane Care and Use of Laboratory Animals (US Department of Health and Human Services, Washington, DC, 1986; amended 2002).

\title{
Human or mouse? Practice makes perfect
}

T he IACUC must assure the personnel performing procedures in the IACUC submissions are trained and qualified. This can be attained by:

1. Proper training of personnel, allowing easy access to training modalities

2. Consultations by subject matter experts and more rigorous review of IACUC protocols by trained and engaged IACUC members

3. Additional questions added to IACUC submission form (which may include criteria for external consultants)

4. A PAM (post approval monitoring) program to encourage investigators to improve their data outcomes and avoid unrealized risks.

Following such considerations could have helped Great Eastern University avoid the current situation. A consultation, or pre-review, of the IACUC application by a veterinarian or expert designated by the Attending Veterinarian (AV) may have taken the lack of expertise with the species into consideration and prompted the IACUC to request more information regarding Schwartz' experience. As stated in ref. above, "The IACUC assumes responsibility on behalf of the institution and thereby has the function of overseeing the training program." At a minimum, there could have been additional assurance that proper methods of euthanasia were followed for compromised animals.

White, the principal investigator (PI) could have addressed the lack of expertise of the surgeon by accounting for animals for training use by Schwartz within the IACUC application. This would have assured the IACUC that proper precautions would be taken for this novel procedure in the mice, including humane endpoints.

The IACUC could have requested to see curriculum vitae (CV) of the surgeon's credentials and experience when reviewing the IACUC application. This may have alerted the IACUC to the lack of experience with the intended species for use on this protocol. A requirement for $\mathrm{CV}$ submission when listing non-departmental personnel on protocols for surgical procedures would forewarn the IACUC for future submissions.

Additional questions in the IACUC application form template regarding experience (or training) would be helpful for the IACUC to assure if the "personnel conducting procedures on the species being maintained or studied will be appropriately qualified and trained in those procedures," as indicated in the Animal Welfare Act and Regulations ${ }^{2}$. Additionally, the Guide for the Care and Use of Laboratory Animals states, "Researchers conducting surgical procedures must have appropriate training to ensure good surgical technique is practiced-that is, asepsis, gentle tissue handling, minimal dissection of tissue, appropriate use of instruments, effective hemostasis, and correct use of surgical materials and patterns." ${ }^{3}$

In response to the deaths of the animals, the AV (or one of the school's veterinarians) could observe the surgery to assure the IACUC that proper veterinary oversight is conducted for the welfare of the animals. If the procedure is conducted according to the IACUC submission without incident, the AV (or school veterinarian) may communicate the assurance to the IACUC for the surgeries to be conducted by Schwartz. If the procedure is not conducted without incident, the AV (or school veterinarian) would halt the surgeries, taking proper precautions for clinical care and animal welfare. The AV (or school veterinarian) would communicate concerns to the IACUC to halt the IACUC application. Further explanation and follow-up between the IACUC and White should take place.

Further follow up on this IACUC application could be conducted as a PAM since there is a post approval monitoring program at Great Eastern University, if through further discussions it is deemed appropriate to continue with the IACUC submission.

Lisa Stanislawczyk

Bristol-Myers Squibb, New Brunswick, NJ, USA.

e-mail: Lisa.Stanislawczyk@bms.com

Published online: 23 October 2019

https://doi.org/10.1038/s41684-019-0419-2

References

1. The Care and Feeding of an IACUC; The Organization and Management of an Institutional Animal Care and Use Committee, Second Edition, (CRC Press, Taylor \& Francis Group, 2015).

2. Animal Welfare Regulations Part 2, Subsection C. $\$ 2.31$ Institutional Animal Care and Use Committe.

3. Institute for Laboratory Animal Research. Guide for the Care and Use of Laboratory Animals 8th edn. pg. 15-17, 25-26 (National Academies Press, Washington, DC, 2011). 